\title{
WORKING PAPERS EM LINGÜÍSTICA
}

\author{
VOLUME 11 (1) \\ JAN. JUN. - 2010
}

\begin{abstract}
Autores deste número
Ana Paula Kuczmynda Silveira (ana_paula_k.silveira@terra.com.br)

Antonio José de Pinho (antoniojp@hotmail.com)

Bruno Cardoso (brunoletras_ufsc@hotmail.com)

Edair Gorski (gorski@cce.ufsc.br)

Eloara Tomazoni (eloara_tomazoni@hotmail.com)

Michelle Donizeth Euzébio (michelledoni@yahoo.com.br)

Tatiana Schwochow Pimpão (tatiana.pimpao@hotmail.com)
\end{abstract}

\author{
Editoras \\ Izete Lehmkuhl Coelho (izete@cce.ufsc.br) \\ Rosângela Hammes Rodrigues (hammes@cce.ufsc.br) \\ Coordenadora do Programa de Pós-Graduação em Linguística \\ Universidade Federal de Santa Catarina
}

\author{
Equipe Editorial \\ Rosangela Hammes Rodrigues \\ Izabel Christine Seara \\ Izete Lehmkuhl Coelho \\ Mary Elizabeth Cerutti Rizzatti \\ Felício Wessling Margotti
}




\section{Conselho Editorial}

Aline Cacilda Koteski Emilio - UEPG (PR)

Adriana Fischer - UNIFEBE (Brusque/SC)

André Berri - UFSC

Carlos Mioto - UFSC

Celina Maria Ramos Arruda Macedo - UFSC

Clarice Nadir von Borstel - UNIOESTE (PR)

Edair Maria Gorski - UFSC

Fabio Luiz Lopes da Silva - UFSC

Heronides Maurílio de Melo Moura - UFSC

Josias Ricardo Hack - UFSC

Leonor Scliar Cabral - UFSC

Luizete Guimarães Barros - UFSC

Magdiel Medeiros Aragão Neto - UFAM

Maria Inêz Probst Lucena - UFSC

Maria Izabel de Bortoli Hentz - UFSC

Maria Marta Furlanetto - UNISUL (SC)

Márluce Coan - UFC

Maurício Eugênio Maliska - UNISUL (SC)

Monica Mano Trindade - UFPB

Nelita Bortolotto - UFSC

Pedro de Souza - UFSC

Renato Miguel Basso - UFSC

Roberta Pires de Oliveira - UFSC

Ronald Taveira da Cruz - UFPI

Rosely Xavier - UFSC

Sandro Braga - UNISUL (SC)

Simone Bueno Borges da Silva - UFBA

Tarcisio de Arantes Leite - UFSC

Teresinha de Moraes Brenner - UFSC

Werner Heidermann - UFSC

(Catalogação na fonte pela DECTI da Biblioteca da UFSC)

Working papers in lingüística / Programa de Pós-graduação em Lingüística. Universidade Federal de Santa Catarina. v.11, n.1. (2010)

Florianópolis : Universidade Federal de Santa Catarina, Pós-graduação em Lingüística, 2010

Semestral

Anual 1997-2004; Semestral 2008 -

Resumo em português e inglês

A partir de maio de 2008, disponível no portal de periódicos da UFSC em: http://www.periodicos.ufsc.br

pISSN 1516-1464

eISSN 1984-8420

1. Lingüística. 2. Linguagem. 3. Língua Portuguesa I. Universidade Federal de Santa Catarina. Pós-graduação em Lingüística. Curso de Letras 